\title{
Mutazioni del gene USP8 che causano guadagno di funzione nella malattia di Cushing
}

\author{
Salvatore Cannavò ${ }^{1}$
}

Pubblicato online: 10 aprile 2015

(C) Springer International Publishing AG 2015

Commento a:

Recurrent gain-of-function USP8 mutations in Cushing's disease.

Z.Y. Ma, Z.J. Song, J.H. Chen, Y.F. Wang, S.Q. Li, L.F. Zhou, Y. Mao, Y.M. Li, R.G. Hu, Z.Y. Zhang, H.Y. Ye, M. Shen, X.F. Shou, Z.Q. Li, H. Peng, Q.Z. Wang, D.Z. Zhou, X.L. Qin, J. Ji, J. Zheng, H. Chen, Y. Wang, D.Y. Geng, W.J. Tang, C.W. Fu, Z.F. Shi, Y.C. Zhang, Z. Ye, W.Q. He, Q.L. Zhang, Q.S. Tang, R. Xie, J.W. Shen, Z.J. Wen, J. Zhou, T. Wang, S. Huang, H.J. Qiu, N.D. Qiao, Y. Zhang, L. Pan, W.M. Bao, Y.C. Liu, C.X. Huang, Y.Y. Shi, Y. Zhao.

Cell Research (2015) doi:10.1038/cr.2015.20

Il recentissimo riscontro di mutazioni puntiformi somatiche missenso a carico del gene che codifica per la proteasi ubiquitino-specifica 8 (USP8) in adenomi ipofisari ACTHsecernenti rappresenta una novità di estremo interesse per la comprensione della patogenesi di tali tumori e per le prospettive terapeutiche della malattia di Cushing. Nel primo studio condotto da Reincke et al. su un gruppo eterogeneo di tumori ipofisari, alcune mutazioni puntiformi a carico di tale gene sono state individuate in un terzo dei corticotropinomi, ma in nessuno degli altri tumori ipofisari analizzati, inclusi 2 adenomi ACTH-secernenti di pazienti con sindrome di Nelson [1]. Tali mutazioni, che si localizzano in prossimità del dominio catalitico della proteina, comportano una ridotta ubiquitinizzazione del recettore per l'Epidermal Growth Factor (EGFR), con sua conseguente attivazione costitutiva

S. Cannavò

cannavos@unime.it

1 Dipartimento di Medicina Clinica e Sperimentale, Università di Messina, Messina, Italia che induce la trascrizione di proopiomelanocortina (POMC) e, a sua volta, un'aumentata sintesi e secrezione di ACTH. Secondo questo studio, tuttavia, le mutazioni di USP8 non comporterebbero una significativa modificazione dell' attività proliferativa delle cellule neoplastiche, per cui gli adenomi ACTH-secernenti con tali mutazioni presentano piccole dimensioni e basso indice proliferativo [1].

Lo studio di Ma et al. conferma ed estende i risultati dello studio precedente. In questo nuovo studio, infatti, 17 differenti mutazioni somatiche di USP8 sono state individuate nel $62,5 \%$ di 120 corticotropinomi, mentre nessuna mutazione è stata identificata in 150 adenomi ipofisari non ACTH-secernenti. La presenza della mutazione comporterebbe un effetto protettivo sulla degradazione lisosomiale dell'EGFR, che si traduce in un'elevata espressione e accumulo di EGFR all'interno della cellula. A ciò corrisponde un' aumentata espressione di POMC mRNA, che codifica per il precursore dell'ACTH. In linea con tali evidenze, i pazienti portatori di tali mutazioni somatiche si caratterizzano per livelli più elevati di ACTH circolante, sebbene il diametro dell'adenoma sia significativamente più piccolo.

In futuro, l'inattivazione del gene USP8 con mutazione che induce guadagno di funzione, o direttamente dell'EGFR, potrebbe rappresentare un potenziale target terapeutico per i pazienti con malattia di Cushing.

\section{Bibliografia}

1. Reincke M, Sbiera S, Hayakawa A et al (2015) Mutations in the deubiquitinase gene USP8 cause Cushing's disease. Nat Genet 47:31-38 